\title{
Multimodal Pain Management With Adductor Canal Block Decreases Opioid Consumption Following Total Knee Arthroplasty
}

\author{
Neil Thomas Soehnlen, MD; Eric Donald Erb, MD; Eric McKee Kiskaddon, MD; Uthona Green, DNP; \\ Andrew Froehle, PhD; and Anil Krishnamurthy, MBBS
}

\begin{abstract}
Background: Ease of access to opioids in the perioperative period is a risk factor for subsequent opioid misuse. The purpose of this study was to quantify a decrease in opioid consumption following implementation of a new analgesic protocol after total knee arthroplasty (TKA).

Methods: A retrospective cohort study was performed analyzing patients who underwent TKA at a US Department of Veterans Affairs medical center. Patients were divided into 2 groups by multimodal analgesic regimen: Analgesia with intraoperative general anesthesia, a patient-controlled analgesia pump, and oral opioids (control group) or analgesia with intraoperative spinal anesthesia, a multimodal medication regimen, and an adductor canal block (protocol group).
\end{abstract}

Results: A total of 533 TKAs were included. The mean (SD) IV morphine equivalent dose (MED) requirement was 178.2 (98.0) for the control and 12.0 (24.6) for the protocol group $(P<.001)$. Total mean (SD) opioid MED requirement was 241.7 (120.1) for the control group and $74.8(42.7)$ for the protocol group $(P<.001)$. The protocol group required only $6.7 \%$ of the IV opioids and the control group $30.9 \%$. No difference in oral opioid requirements was found $(P=.85)$. The control group required more opioid refills at the first postoperative visit $(P<.001)$.

Conclusions: The described analgesic protocol resulted in significant decreases in IV and total opioid requirement, and lower rates of opioid prescriptions at the first postoperative visit. These findings demonstrate a decrease in opioid utilization with modern perioperative analgesia protocols and reinforce multiple recommendations to decrease opioid exposure and access.
Author affiliations can be found at the end of the article.

Correspondence:

Eric Erb (rerb3@gmail.com)

Fed Pract. 2021;38(12). Published online December 12. doi:10.12788/fp.0209

\footnotetext{
fied
}

$\longrightarrow$ o ase of access to opioids in the perioperative period is a risk factor for opioid misuse and has been identified as a strong risk factor for heroin use. ${ }^{1,2}$ Three-quarters of today's heroin users were introduced to opioids through prescription medications. ${ }^{2}$ The United States accounts for about $80 \%$ of the global opioid supply consumption, and deaths from opioid overdose are increasing: 70,630 deaths in 2019 alone. ${ }^{3,4}$

The Centers for Disease Control and Prevention (CDC) has called for changes in opioid prescribing. The American Academy of Orthopaedic Surgeons (AAOS) also has published an information statement with strategies to decrease opioid misuse and abuse. .,6 $^{-1}$ Arthroplasty surgeons have recently focused on decreasing use of opioids in total knee arthroplasty (TKA), a procedure traditionally associated with high levels of opioid consumption and historical reliance on opioid monotherapy for postoperative analgesia. ${ }^{7,8}$ From a clinical perspective, prolonged postoperative opioid use contributes to poorer surgical outcomes due to increased risk of complications, including stiffness, infection, and revision TKA. ${ }^{9}$

Multimodal pain regimens are increasingly being used to control postoperative pain as data supports their efficacy. ${ }^{10,11}$ Pre- vious studies have found that simultaneous modulation of multiple pain pathways decreases narcotics consumption and improves patient outcomes. ${ }^{12,13}$ Along with other adjuvant therapies, peripheral nerve blocks, such as adductor canal block (ACB) and femoral nerve block (FNB), have been used to decrease postoperative pain. ${ }^{14}$ Studies have shown that ACB has fewer complications and shorter functional recovery times compared with FNB. ${ }^{15,16}$ The distribution of the ACB excludes the femoral nerve, thus preserving greater quadriceps strength while providing equivalent levels of analgesia compared with FNB. ${ }^{15,17,18}$ The ACB has shown decreased near-fall events and improved balance scores in the immediate postoperative period. ${ }^{19}$

Our study analyzed opioid consumption patterns of TKA patients from a US Department of Veterans Affairs (VA) medical center before and after the institution of a multimodal analgesic protocol using ACB. The primary purpose of this study was to determine whether a protocol that included intraoperative spinal anesthesia with a postoperative multimodal analgesic regimen and ACB was associated with a decreased postoperative opioid requirement when compared with patients who received intraoperative general anesthesia and a traditional opioid regimen. Secondary outcomes included the 
effect of opioid consumption on range of motion on postoperative day (POD) 1 and number of opioid prescriptions written at the first postoperative clinic visit.

\section{METHODS}

Approval for the study was obtained from the institutional review board at the Dayton Veterans Affairs Medical Center (DVAMC) in Ohio. A retrospective chart review was performed to collect data from all patients undergoing TKA at DVAMC from June 1, 2011, through December 31, 2015. Exclusion criteria included multiple surgeries in the study time frame, documented chronic pain, allergy to local anesthetics, daily preoperative use of opioids, and incomplete data in the health record.

All surgeries were performed by 2 staff arthroplasty surgeons at a single VAMC. All patients attended a preoperative visit where a history, physical, and anesthesia evaluation were performed, and watched an educational video detailing surgical indications and postoperative rehabilitation. All surgeries were performed with tourniquets and a periarticular injection was performed at the conclusion of each case. Surgeon 1 treatment of choice was $10 \mathrm{~mL} 0.5 \%$ bupivacaine, whereas surgeon 2 performed a posterior capsular injection of $30 \mathrm{~mL} 0.25 \%$ bupivacaine and a periarticular injection of $30 \mathrm{mg}$ ketorolac in $10 \mathrm{~mL} 0.25 \%$ bupivacaine with epinephrine.

Prior to August 2014, general endotracheal anesthesia was used intraoperatively. A patient-controlled analgesia (PCA) pump of morphine or hydromorphone and additional oral oxycodone or hydrocodone was used for postoperative pain. PCA pumps were patient dependent. In the control group, 245 patients received the morphine PCA while 61 received the hydromorphone PCA. Morphine PCA dosing consisted of 1 -mg doses every 10 minutes with potential baseline infusion rates of 0.5 to $1.0 \mathrm{mg} / \mathrm{h}$ and a 4-hour limit of $20 \mathrm{mg}$. Hydromorphone PCA dosing consisted of 0.2 to $0.4-\mathrm{mg}$ doses with a potential continuous dose of 0.2 to $0.4 \mathrm{mg} / \mathrm{h}$ and a 4 -hour limit of $4 \mathrm{mg}$.

In August 2014, a new analgesic protocol was adopted for TKA consisting of intraoperative spinal anesthesia $(0.75 \%$ bupivacaine) with IV sedation (propofol), a postoperative multimodal analgesic regimen, an ACB performed in the postanesthe-
TABLE 1 Preoperative Demographic Characteristics

\begin{tabular}{lccc} 
Variables & $\begin{array}{c}\text { Control } \\
(\mathbf{n}=\mathbf{3 0 6})\end{array}$ & $\begin{array}{c}\text { Protocol (n } \\
\mathbf{=} \mathbf{2 2 7})\end{array}$ & $\begin{array}{c}\boldsymbol{P} \\
\text { value }^{\mathbf{a}}\end{array}$ \\
\hline Age, mean (SD), y & $63.5(8.6)$ & $65.0(8.1)$ & .04 \\
\hline Sex, male/female, \% & $91.2 / 8.8$ & $93.0 / 7.0$ & .52 \\
\hline Body mass index, mean (SD) & $31.5(4.9)$ & $32.2(4.0)$ & .09 \\
\hline Range of motion, ${ }^{\circ}$ (SD) & & & \\
Maximum knee flexion & $111.0(15.0)$ & $111.3(12.6)$ & .84 \\
Maximum knee extension & $5.0(6.4)$ & $4.0(5.7)$ & .05 \\
Knee total arc & $106.0(17.9)$ & $107.3(15.1)$ & .38 \\
\hline Comorbid conditions, \% & & & \\
Diabetes mellitus type 1 & 2.6 & 1.3 & .37 \\
Diabetes mellitus type 2 & 25.8 & 32.2 & .12 \\
Coronary artery disease & 16.3 & 19.8 & .31 \\
Chronic obstructive pulmonary disease & 5.9 & 13.7 & .002 \\
Chronic kidney disease & 2.6 & 3.5 & .61 \\
Deep vein thrombosis history & 2.0 & 4.0 & .19 \\
Pulmonary embolism history & 1.3 & 1.8 & .73 \\
\hline Preoperative emergency department & & & \\
visits, mean (SD) & $0.53(1.3)$ & $0.45(1.0)$ & .48 \\
\hline
\end{tabular}

${ }^{a}$ Between-groups differences using independent samples using $t$ tests for continuous data and Fisher exact tests for discrete data.

sia care unit (PACU), and opioids as needed (protocol group). The ACB catheter was a $0.5 \%$ ropivo caine hydrochloride injection. It was attached to a local anesthetic fixed flow rate pump that administers $0.5 \%$ ropivacaine without epinephrine at $8 \mathrm{~mL} / \mathrm{h}$ and was removed on POD 5 by the patient. The multimodal medication regimen included IV ketorolac 15 mg every 6 hours for 3 doses, gabapentin $300 \mathrm{mg}$ every 8 hours, acetaminophen 975 mg every 8 hours, meloxicam $7.5 \mathrm{mg}$ daily, tramadol $50 \mathrm{mg}$ every 6 hours, oxycodone $5 \mathrm{mg} 1$ to 2 tabs every 4 hours as needed, and IV hydromorphone $0.5 \mathrm{mg}$ every 4 hours as needed for breakthrough pain.

Preoperative demographic characteristics were collected (Table 1). Data on all IV and oral opioid requirements were collected for both groups, converted to morphine milligram equivalents (MME), and a total morphine equivalent dose (MED) was

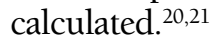

In April 2015, a separate protocol change occurred at the DVAMC with the goal of discharge on POD 1. To standardize outcomes before and after this change, data collection regarding opioid requirements was concluded at midnight on POD 1 . If a 
TABLE 2 Perioperative Characteristics

\begin{tabular}{lccc} 
Variables & Control $(\mathbf{n = 3 0 6 )}$ & Protocol $(\mathbf{n = 2 2 7})$ & $\boldsymbol{P}$ value $^{\mathbf{a}}$ \\
\hline Surgeon 1/2, \% & $55.6 / 44.4$ & $52.4 / 47.6$ & .48 \\
\hline Tourniquet time, mean (SD) & $68.1(13.8)$ & $69.8(12.4)$ & .13 \\
\hline $\begin{array}{l}\text { Length of stay, mean (SD), d } \\
\text { aBetween-groups differences using independent samples using t tests for continuous } \\
\text { data and Fisher exact tests for discrete data. }\end{array}$ & $2.5(1.3)$ & $1.4(0.7)$ & .001 \\
\end{tabular}

patient was discharged before midnight on POD 1, opioid requirement through the time of discharge was collected. All surgeries were performed in the morning to early afternoon; however, specific surgical times were not collected. Patients were also evaluated by a physical therapist on POD 0 , and maximal knee flexion and extension were measured on POD 1. Patients were discharged with prescriptions for oxycodone/ acetaminophen and tramadol and were seen 3 weeks later for their first postoperative visit. Opioid refills at the first postoperative visit were recorded.

All statistical analyses were performed in SAS 9.4 with significance set to $\alpha=0.05$. Between-groups differences in preoperative and perioperative characteristics as well as postoperative outcomes were analyzed using independent samples $t$ tests for continuous variables and Fisher exact tests for dichotomous discrete variables. Where groups differed for a pre- or perioperative variable, linear mixed models analysis was used to determine whether IV, oral, and total MEDs were significantly affected by the interaction between the pre- or perioperative variable with analgesia group. For refills at the postoperative visit, the effects of pre- or perioperative differences were tested using $\chi^{2}$ tests. Effect sizes for outcome variables were estimated using Cohen $d$ and probability of superiority $(\Delta)$ for continuous variables, and relative risk (RR) in the case of discrete variables. ${ }^{22}$

\section{RESULTS}

During the study period from June 1, 2011, through December 31, 2015, 533 eligible TKAs were performed, 306 in the control group and 227 in the protocol group. The groups had similar sex distribution; body mass index; knee range of motion; diagnoses of diabetes mellitus, coronary artery disease, and chronic kidney disease; and history of deep vein thrombosis (DVT) or pulmonary embolism $(P \geq .05)$. The protocol group was significantly older $(P=.04)$ and had a significantly higher rate of chronic obstructive pulmonary disease $(C O P D)(P=.002)$. There were no significant differences between number of procedures performed by surgeon $(P=.48)$ or total tourniquet time $(P=.13)$ (Table 2). Mean (SD) length of stay was significantly greater in the control group compared with the protocol group (2.5 [1.3] vs 1.4 [0.7] days, $P<.001$ ).

Figure 1 shows the distributions of each type of opioid used. Compared with the control group, the protocol group had a significantly lower mean (SD) IV opioid use: 178.2 (98.0) MED vs 12.0 (24.6) MED $(P<.001 ; d=2.19 ; \Delta=0.94)$ and mean (SD) total opioid use: 241.7 (120.1) MED vs 74.8 (42.7) $\mathrm{MED}(P<.001 ; d=1.76$; $\Delta=0.89)$. Mean (SD) oral opioid use did not differ between groups (control, 63.6 [45.4] MED; protocol, 62.9 [31.4] MED; $P=.85 ; d=0.02 ; \Delta=0.51$ ). A significantly lower percentage of patients in the protocol group received additional opioids at the 3-week follow-up when compared to the control group: $46.7 \%$ vs $61.3 \%$, respectively $(P<.001$; RR, 0.76; 95\% CI, 0.65-0.90).

There were no significant differences in postoperative mean (SD) maximum knee flexion (control, 67.2 [15.7 $]^{\circ}$; protocol, $67.8[19.2]^{\circ} ; P=.72 ; d=0.03 ; \Delta=0.51$ ) or mean (SD) total flexion/extension arc (control, 66.2 [15.9] $]^{\circ}$; protocol, 67.9 [19.4 $^{\circ}$; $P=.32 ; d=0.10 ; \Delta=0.53)$. Mean (SD) postoperative maximum knee extension was significantly higher in the protocol group compared with the control group $\left(-0.1[2.1]^{\circ}\right.$ vs $\left.1.0[3.7]^{\circ} ; P<.001 ; d=0.35 ; \Delta=0.60\right)$. More patients in the protocol group (92.5\%) were discharged to home compared with the control group $(86.6 \%)(P=.02 ; \mathrm{RR}, 1.07$; 95\% CI, 1.01-1.13).

Because age and rates of COPD differed between groups, sensitivity analyses were conducted to determine whether these variables influenced postoperative opioid use. The relationship between age and group was significant for IV $(P<.001)$ and total opioid use $(P<.001)$. Younger patients received higher MED doses than older patients within the control group, while dosages were fairly consistent regardless of 


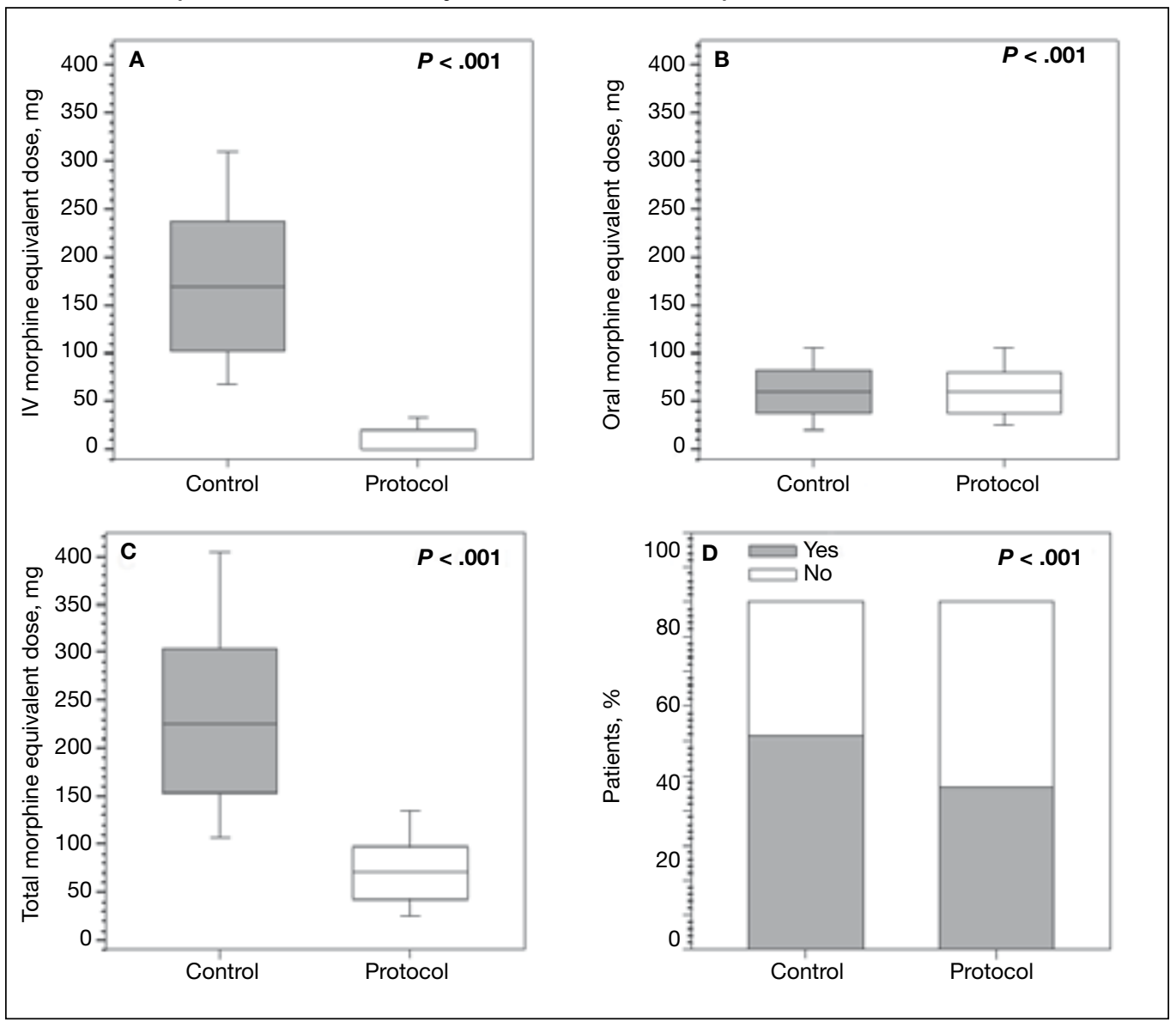

age in the protocol group (Figure 2). There was no significance in age interaction effect with regard to oral opioids $(P=.83)$ nor opioid refills at 3-week follow-up $(P=.24)$.

The sensitivity analysis for COPD found that a diagnosis of COPD did not significantly influence utilization of IV opioids $(P=.10)$, or total opioids $(P=.68)$. There was a significant interaction effect for oral opioids (Figure 3). Patients in the control group with COPD required significantly higher mean (SD) oral opioids than patients without COPD (91.5 [123.9] MED and 62.0 [36.0] MED, respectively; $P=.03$ ). In the control group, the $\chi^{2}$ test was significant regarding opioid prescription refills at the 3 -week visit $(P=.004)$ with $62.4 \%$ of patients with COPD requiring refills vs $44.4 \%$ without COPD $(P=.004)$. There was no difference in refills in the protocol group (46.4\% vs $48.4 \%$ ).

Finally, 2-sided independent samples $t$ test evaluated total MED use between the 2 surgeons. There was no difference in total MED per patient for the surgeons. In the control group, mean (SD) total MED for surgeon 1 was 232.9 (118.7) MED vs 252.8 (121.5) MED for surgeon $2(P=.18)$. In the protocol group, the mean (SD) total MED was 72.5 (43.2) and 77.4 (42.1) for surgeon 1 and surgeon 2 , respectively $(P=.39)$.

\section{DISCUSSION}

Coordinated efforts with major medical organizations are being made to decrease opioid prescriptions and exposure. ${ }^{5,6}$ To our knowledge, no study has quantified a decrease in opioid requirement in a VA population after implementation of a protocol that includes intraoperative spinal anesthesia and a postoperative multimodal analgesic regimen including ACB after TKA. The analgesic protocol described in this study aligns with recommendations from both 
FIGURE 2 Effect of Age on Opioid Outcomes

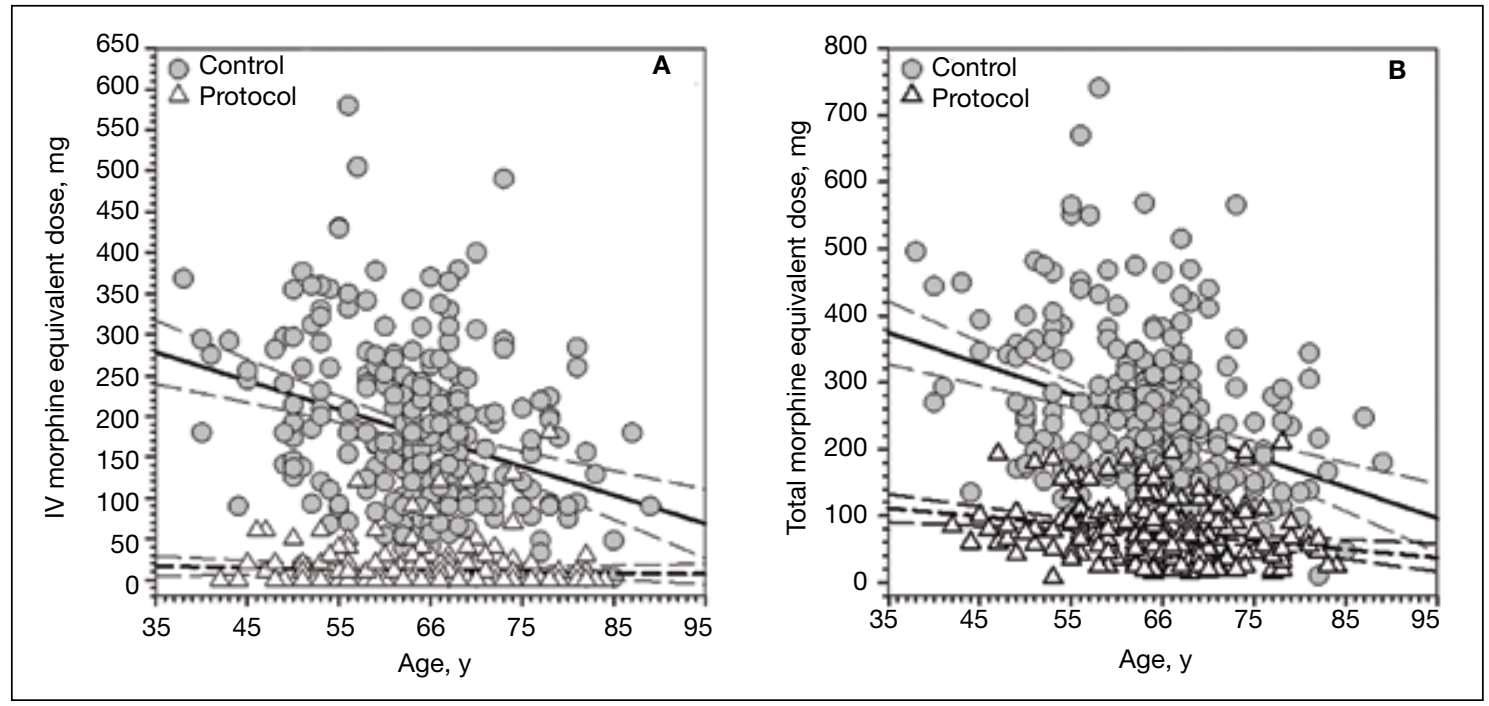

the CDC and the AAOS to decrease opioid use and misuse by maximizing nonopioid medications and limiting the size and number of opioid prescriptions. However, public and medical opinion of opioids as well as prescribing practices have changed over time with a trend toward lower opioid use. The interventions, as part of the described protocol, are a result of these changes and attempt to minimize opioid use while maximizing postoperative analgesia.

Our data showed a significant decrease in total opioid use through POD 1, IV opioid use, and opioid prescriptions provided at the first postoperative visit. The protocol group used only $6.7 \%$ of the IV opioids and $30.9 \%$ of the total opioids that were used by the control group. The substantial difference in IV opioid requirement, 166.2 MED, is equivalent to $8 \mathrm{mg}$ of IV hydromorphone or $55 \mathrm{mg}$ of IV morphine. The difference in total opioid requirement was similar at 166.9 MED, equivalent to $111 \mathrm{mg}$ of oral oxycodone.

Decreasing opioid use has the additional benefit of improving outcomes, as higher doses of opioids have been associated with increased length of stay, greater rates of DVT, and postoperative infection. ${ }^{23}$ These complications occurred in a stepwise manner, suggesting a dose-response gradient that makes the sizable decrease noted in our data of greater relevance. ${ }^{23}$ While the adverse effects (AEs) of opioids are well known, there are limited data on opioid dosing and its effect on perioperative outcomes. ${ }^{23}$

A significant decrease in the percentage of patients receiving an opioid prescription at the first postoperative visit suggests a decrease in the number of patients on prolonged opioids after TKA with implementation of modern analgesic modalities. The duration of postoperative opioid use has been found to be the strongest predictor of misuse, and each postoperative refill increases the probability of misuse by $44 \% .{ }^{24}$ In addition, opioid use for $>3$ months after TKA is associated with increased risk of periprosthetic infection, increased overall revision rate, and stiffness at 1 year postoperatively. ${ }^{9}$ While not entirely under the control of the surgeon, measures to decrease the number of postoperative opioid refills may lead to a decrease in opioid misuse.

In the control group, older patients tended to receive less opioids. This is likely due to physiologic changes in opioid metabolism associated with aging, including decreased renal and hepatic opioid metabolism and alterations in overall body composition that increase relative potency and duration of action of opioids in a geriatric population. ${ }^{25,26}$ No difference in opioid use by age was found for the protocol group.

Patients in the protocol group demonstrated significantly greater maximal knee extension on POD 1 compared with the control group. No difference in maximal flexion was found. This difference in extension may 
FIGURE 3 Interaction Effect of COPD and Group on Opioid Use

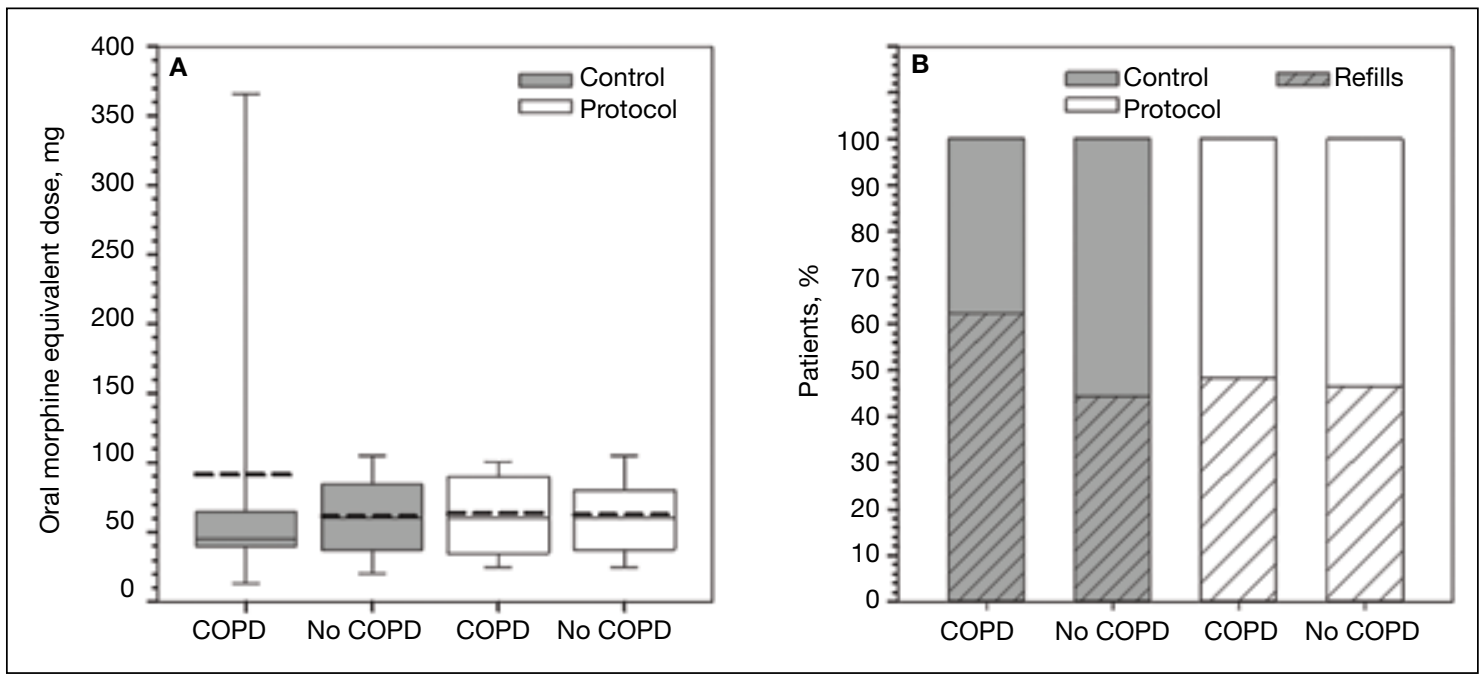

Abbreviation: COPD, chronic obstructive pulmonary disease.

partially be explained by the use of an ACB. One benefit of ACB is greater quadriceps strength and fewer near-fall events when compared with FNB. ${ }^{15,19}$

Our results corroborate the findings of similar studies. A randomized controlled trial comparing a multimodal analgesic regimen with a periarticular injection without a postoperative ACB to a hydromorphone PCA revealed a significant decrease in opioid use in the multimodal analgesic group. ${ }^{27}$ Along with lower opioid requirements, the multimodal analgesic group had lower visual analog scale pain scores, fewer AEs, faster progression to physical therapy milestones, and higher satisfaction. ${ }^{27}$ Recent guidelines from the French Society of Anaesthesia and Intensive Care Medicine recommend against the use of gabapentin as a method of postoperative pain control. However, this specifically refers to the preoperative administration of gabapentin. This same set of guidelines later cites a high level of evidence suggesting patients undergoing arthroplasty benefit more from gabapentinoids. ${ }^{28}$ Multiple analgesic protocols that include gabapentin as a part of a multimodal approach have been shown to have positive results. 13,29

In our study, patients receiving the multimodal analgesic regimen were significantly more likely to be discharged home rather than to postacute care facilities, which have been associated with increased rates of major complications, 30-day readmission, and 30-day reoperation. ${ }^{30,31}$ In addition, discharge to an inpatient rehabilitation or skilled nursing facility has not been found to result in higher functional outcomes, despite $\$ 3.2$ billion spent yearly on rehabilitation services after primary TKA. ${ }^{32,33}$

A component of our described analgesic protocol included spinal anesthesia intraoperatively. The differences between groups regarding anesthesia type can be attributed to this protocol change. A significantly greater percentage of patients in the protocol group received spinal anesthesia, while more patients in the control group received general anesthesia. While patients who received spinal anesthesia may have enhanced analgesia in the immediate postoperative period, no differences in opioid outcomes were seen based on anesthesia type. Known benefits of intraoperative spinal anesthesia include decreased perioperative blood loss and a smaller decrease in hemoglobin postoperatively, as well as lower rates of in-hospital complications, including pulmonary embolism, pneumonia, cerebrovascular events, and acute renal failure. ${ }^{34}$

\section{Limitations}

A number of limitations of this study should be noted. One was a protocol change regarding length of stay, which occurred during the study period and resulted in a significantly shorter length of stay in the protocol group. As a result, opioid 
use data were analyzed only through midnight at the end of POD 1. Patients who were discharged on POD 1 did not have opioid use data available for the full duration of the first POD, which may exaggerate the decrease in opioid requirements, as opioids used after discharge but prior to midnight on POD 1 were not recorded. However, opioids taken at home are oral with a low MME compared with IV opioids received by hospitalized patients in the control group. In addition, if taken as prescribed, patients at home would only have enough time to take a few doses of opioids prior to the midnight cutoff. We do not believe this difference in time of opioid use meaningfully affected the data. An additional limitation includes the variability between periarticular injections between surgeons. While the percentage of patients that received injections from surgeon 1 vs surgeon 2 were similar, it cannot be ruled out as a potential confounding factor. Other limitations include a lack of pain scores to compare subjective pain ratings, the retrospective nature of the study, and a largely homogenous male VA population.

\section{CONCLUSIONS}

Ease of access to opioids is a risk factor for opioid abuse, which itself is a risk factor for subsequent heroin use. ${ }^{1,2}$ The CDC and AAOS have thus published recommendations regarding opioid prescribing practices to decrease opioid use and abuse..$^{5,6}$ Our described protocol, which aligns with these recommendations, resulted in a significant decrease in IV opioid requirement, total opioid requirement, and lower rates of opioid prescriptions provided at the first postoperative visit. These promising findings demonstrate a lower percentage of patients on long-term opioids after TKA and a significantly decreased cumulative opioid exposure.

\section{Author affiliations}

Neil Soehnlen, Eric Erb, Eric Kiskaddon, and Anil Krishnamurthy are Orthopaedic Surgeons; Uthona Green is an Orthopaedic Advanced Practice Nurse; all at Dayton Veterans Affairs Medical Center in Ohio. Andrew Froehle is an Associate Professor; Neil Soehnlen and Eric Erb are Residents in the Department of Orthopaedic Surgery; Anil Krishnamurthy is the Program Director of Orthopaedic Surgery; all at Wright State University. Eric Kiskaddon was a Resident in the Department of Orthopaedic Surgery at Wright State University at the time of this study and is now a Fellow in
Adult Reconstruction at Ohio State University Hospital in Columbus.

\section{Author disclosures}

The authors report no actual or potential conflicts of interest or outside sources of funding with regard to this article.

\section{Disclaimer}

The opinions expressed herein are those of the authors and do not necessarily reflect those of Federal Practitioner, Frontline Medical Communications Inc., the US Government, or any of its agencies. This article may discuss unlabeled or investigational use of certain drugs. Please review the complete prescribing information for specific drugs or drug combinations - including indications, contraindications, warnings, and adverse effects-before administering pharmacologic therapy to patients.

\section{Ethics and consent}

This article does not contain any studies with human participants or animals performed by any of the authors. Full institutional review board approval for human data was obtained through both Wright State University as well as the Dayton Veterans Affairs Medical Center institutional review boards. Informed consent was not required for this consent-exempt study.

\section{References}

1. Lankenau SE, Teti M, Silva K, Jackson Bloom J, Harocopos A, Treese M. Initiation into prescription opioid misuse amongst young injection drug users. Int J Drug Policy. 2012;23(1):37-44. doi:10.1016/j.drugpo.2011.05.014

2. Jones CM. Heroin use and heroin use risk behaviors among nonmedical users of prescription opioid pain relievers - United States, 2002-2004 and 2008-2010. Drug Alcohol Depend. 2013;132(1-2):95-100. doi:10.1016/j.drugalcdep.2013.01.007

3. Manchikanti L, Singh A. Therapeutic opioids: a ten-year perspective on the complexities and complications of the escalating use, abuse, and nonmedical use of opioids. Pain Physician. 2008;11(suppl 2):S63-S88.

4. Seth P, Scholl L, Rudd RA, Bacon S. Overdose deaths involving opioids, cocaine, and psychostimulants - United States, 2015-2016. MMWR Morb Mortal Wkly Rep. 2018;67(12):349-358. Published 2018 Mar 30. doi:10.15585/mmwr.mm6712a1

5. Dowell D, Haegerich TM, Chou R. CDC Guideline for Prescribing Opioids for Chronic Pain-United States, 2016. JAMA. 2016;315(15):1624-1645. doi:10.1001/jama.2016.1464

6. American Academy of Orthopaedic Surgeons. Information statement: opioid use, misuse, and abuse in orthopaedic practice. Published October 2015. Accessed November 12, 2021. https://aaos.org/globalassets/about /bylaws-library/information-statements/1045-opioid-use -misuse-and-abuse-in-practice.pdf

7. Hernandez NM, Parry JA, Taunton MJ. Patients at risk: large opioid prescriptions after total knee arthroplasty. J Arthroplasty. 2017;32(8):2395-2398. doi:10.1016/j.arth.2017.02.060

8. Gerner P, Poeran J, Cozowicz C, Mörwald EE, Zubizarreta $\mathrm{N}$, Mazumdar M, Memtsoudis SG, Multimodal pain management in total hip and knee arthroplasty: trends over the last 10 years. Abstract presented at: American Society of Anesthesiologists Annual Meeting; October 21, 2017; Boston, MA.

9. Cancienne JM, Patel KJ, Browne JA, Werner BC. Narcotic use and total knee arthroplasty. J Arthroplasty. 2018;33(1):113-118. doi:10.1016/j.arth.2017.08.006

10. Moucha CS, Weiser MC, Levin EJ. Current strategies in anesthesia and analgesia for total knee arthroplasty. J Am Acad Orthop Surg. 2016;24(2):60-73. doi:10.5435/JAAOS-D-14-00259

11. Wick EC, Grant MC, Wu CL. Postoperative multimodal analgesia pain management with nonopioid analgesics and techniques: a review. JAMA Surg. 2017;152(7):691-697. 
doi:10.1001/jamasurg.2017.0898

12. Lamplot JD, Wagner ER, Manning DW. Multimodal pain management in total knee arthroplasty: a prospective randomized controlled trial. J Arthoplasty. 2014;29(2):329-334. doi:10.1016/j.arth.2013.06.005

13. Golladay GJ, Balch KR, Dalury DF, Satpathy J, Jiranek WA. Oral multimodal analgesia for total joint arthroplasty. J Arthroplasty. 2017;32(9S):S69-S73. doi:10.1016/j.arth.2017.05.002

14. Ardon AE, Clendenen SR, Porter SB, Robards CB, Greengrass RA. Opioid consumption in total knee arthroplasty patients: a retrospective comparison of adductor canal and femoral nerve continuous infusions in the presence of a sciatic nerve catheter. J Clin Anesth. 2016;31:19-26. doi:10.1016/j.jclinane.2015.12.014

15. Li D, Ma GG. Analgesic efficacy and quadriceps strength of adductor canal block versus femoral nerve block following total knee arthroplasty. Knee Surg Sports Traumatol Arthrosc. 2016;24(8):2614-2619. doi:10.1007/s00167-015-3874-3

16. Li D, Yang Z, Xie X, Zhao J, Kang P. Adductor canal block provides better performance after total knee arthroplasty compared with femoral nerve block: a systematic review and meta-analysis. Int Orthop. 2016;40(5):925-933. doi:10.1007/s00264-015-2998-x

17. Horner G, Dellon AL. Innervation of the human knee joint and implications for surgery. Clin Orthop Relat Res. 1994;(301):221-226.

18. Kim DH, Lin Y, Goytizolo EA, et al. Adductor canal block versus femoral nerve block for total knee arthroplasty: a prospective, randomized, controlled trial. Anesthesiology. 2014;120(3):540-550. doi:10.1097/ALN.0000000000000119

19. Thacher RR, Hickernell TR, Grosso MJ, et al. Decreased risk of knee buckling with adductor canal block versus femoral nerve block in total knee arthroplasty: a retrospective cohort study. Arthroplasty Today. 2017;3(4):281-285. Published 2017 Apr 15. doi:10.1016/j.artd.2017.02.008

20. Von Korff M, Saunders K, Thomas Ray G, et al. De facto long-term opioid therapy for noncancer pain [published correction appears in Clin J Pain. 2014 Sep;30(9):830. Korff, Michael Von [corrected to Von Korff, Michael]]. Clin J Pain. 2008;24(6):521-527. doi:10.1097/AJP.0b013e318169d03b

21. Kishner S. Opioid equivalents and conversions: overview. Published January 29, 2018. Accessed November 12 2021. https://emedicine.medscape.com/article/2138678 -overview\#a1

22. Ruscio J, Mullen T. Confidence intervals for the probability of superiority effect size measure and the area under a receiver operating characteristic curve. Multivariate Behav Res. 2012;47(2):201-223. doi:10.1080/00273171.2012.658329

23. Cozowicz C, Olson A, Poeran J, et al. Opioid prescription levels and postoperative outcomes in or- thopedic surgery. Pain. 2017;158(12):2422-2430. doi:10.1097/j.pain.0000000000001047

24. Brat GA, Agniel D, Beam A, et al. Postsurgical prescriptions for opioid naive patients and association with overdose and misuse: retrospective cohort study. BMJ. 2018;360:j5790. Published 2018 Jan 17. doi:10.1136/bmj.j5790

25. Tegeder I, Lötsch J, Geisslinger G. Pharmacokinetics of opioids in liver disease. Clin Pharmacokinet. 1999;37(1):1740. doi:10.2165/00003088-199937010-00002

26. Linnebur SA, O'Connell MB, Wessell AM, et al. Pharmacy practice, research, education, and advocacy for older adults. Pharmacotherapy. 2005;25(10):1396-1430. doi:10.1592/phco.2005.25.10.1396

27. Lamplot JD, Wagner ER, Manning DW. Multimodal pain management in total knee arthroplasty: a prospective randomized controlled trial. J Arthroplasty. 2014;29(2):329334. doi:10.1016/j.arth.2013.06.005

28. Aubrun F, Nouette-Gaulain K, Fletcher D, et al. Revision of expert panel's guidelines on postoperative pain management. Anaesth Crit Care Pain Med. 2019;38(4):405-411. doi:10.1016/j.accpm.2019.02.011

29. Han C, Li XD, Jiang HQ, Ma JX, Ma XL. The use of gabapentin in the management of postoperative pain after total knee arthroplasty: A PRISMA-compliant metaanalysis of randomized controlled trials [published correction appears in Medicine (Baltimore). $2016 \mathrm{Ju}$ 18;95(28):e0916]. Medicine (Baltimore). 2016;95(23):e3883. doi:10.1097/MD.0000000000003883

30. McLawhorn AS, Fu MC, Schairer WW, Sculco PK, MacLean $\mathrm{CH}$, Padgett DE. Continued inpatient care after primary total knee arthroplasty increases 30 -day postdischarge complications: a propensity score-adjusted analysis. J Arthroplasty. 2017;32(9S):S113-S118. doi:10.1016/j.arth.2017.01.039

31. Pelt CE, Gililland JM, Erickson JA, Trimble DE, Anderson $\mathrm{MB}$, Peters $\mathrm{CL}$. Improving value in total joint arthroplasty: a comprehensive patient education and management program decreases discharge to post-acute care facilities and post-operative complications. J Arthroplasty. 2018;33(1):14-18. doi:10.1016/j.arth.2017.08.003

32. Padgett DE, Christ AB, Joseph AD, Lee $Y Y$, Haas SB, Lyman S. Discharge to inpatient rehab does not result in improved functional outcomes following primary total knee arthroplasty. J Arthroplasty. 2018;33(6):1663-1667. doi:10.1016/j.arth.2017.12.033

33. Lavernia CJ, D'Apuzzo MR, Hernandez VH, Lee DJ, Rossi MD. Postdischarge costs in arthroplasty surgery. J Arthroplasty. 2006;21(6 Suppl 2):144-150. doi:10.1016/j.arth.2006.05.003

34. Zhu M, Chen JY, Tan YR, et al. Effects of anesthetic technique on blood loss and complications after simultaneous bilateral total knee arthroplasty. Arch Orthop Trauma Surg. 2015;135(4):565-571. doi:10.1007/s00402-015-2188-8 